\title{
Identification of microRNAs and target genes associated with the development of human intervertebral disc degeneration by bioinformatic analysis and verification
}

\section{Hongze Chang}

Shanghai Yangpu District Central Hospital

\section{Xiaolong Yang}

Shanghai Yangpu District Central Hospital

\section{Kemin You}

Shanghai Yangpu District Central Hospital

Mingwei Jiang

Shanghai Yangpu District Central Hospital

\section{Feng Cai}

Shanghai Yangpu District Central Hospital

\section{Yan Zhang}

Shanghai Yangpu District Central Hospital

\section{Liang Liu}

Shanghai Yangpu District Central Hospital

\section{Hui Liu}

Shanghai Yangpu District Central Hospital

Xiaodong Liu ( $\nabla$ xiaodong.liu@tongji.edu.cn )

Shanghai Yangpu District Central Hospital https://orcid.org/0000-0001-8572-3643

\section{Research article}

Keywords: MicroRNA, Intervertebral disc degeneration, Bioinformatic analysis, Hub gene, Functional pathways

Posted Date: November 14th, 2019

DOI: https://doi.org/10.21203/rs.2.17292/v1

License: (c) (1) This work is licensed under a Creative Commons Attribution 4.0 International License. Read Full License 


\section{Abstract}

Micro RNAs (miRNAs) are widely recognized to play an essential role via target genes in the development of intervertebral disc degeneration( IDD), but the molecular mechanisms remain unclear. To identify the key microRNAs and potential targets during IDD, the Gene Expression Omnibus datasets (GSE19943, GSE63492, and GSE116726) were downloaded.An R package was used to identify differentially expressed miRNAs (DEMs) and four online tools(TargetScan, miRDB, miRTarBase, and DIANA-TarBase) were performed to predict their target genes. Functional enrichment analysis revealed that DEMs gene targets were highly enriched in cell development, cell differentiation, and the p53 and Wnt signaling pathways. we identified 13 hub genes with node degree $\geq 10$ through established a protein-protein interaction (PPI) network. Among them,MAPK8, BMP4, and GSK3B were top 3 highest degree. After constructing the miRNA-target gene-functional analysis network, we found that most hub genes could be regulated by miR-557 and were mainly enriched in cell development, cell differentiation, and Wnt signaling pathway. Further in vitro experiment by qRT-PCR confirmed that miR- 577 was significantly downregulated than the control,whereas miR-516-3p was significantly upregulated. Together, the key microRNA and their target genes identified in this study help us understand the underlying pathogenesis mechanisms in the development of IDD, and provide diagnostic biomarkers and new therapeutic strategies for the treatment of IDD.

\section{Introduction}

Low back pain (LBP) is one of the most common orthopedic diseases; it severely affects human health and expensive medical costs impose a tremendous economic burden on society. The causes of lower back pain are complex, with intervertebral disc degeneration (IDD) is considered as primary pathological changes of LBP[1]. Although various factors are involved in the development of IDD, including aging, excessive weight bearing, trauma, smoking, diabetes and genetic factors [2], the underlying molecular mechanisms has never been satisfactorily explained. Currently, Magnetic resonance imaging (MRI) is the most effective imaging examination of IDD.

However, MRI images only detect IDD in the intermediate or late states instead of the early degenerative period. Therefore, there is a pressing need to understand the precise molecular mechanisms of the IDD process, and thus develop a novel therapeutic target or diagnostic biomarker.

MicroRNAs (miRNA) are a class of short, conserved, noncoding RNAs, which regulate gene expression at the post-transcriptional level by blocking mRNA translation or degrading mRNA via binding to the 3'untranslated regions ( 3 '-UTRs) of target mRNAs. They are fundamental gene regulators that control proliferation, differentiation, and apoptosis during development [3-5]. It has been reported that miRNAs affect the development and progression of IDD by regulating target genes[6, 7] . Furthermore, Several studies suggested that miR-155, miR-141 and miR-221 might be prospective targets for the diagnosis and treatment of IDD[8-10]. However, to date, the function and mechanism of miRNAs in IDD development remains to be elucidated. 
In recently years, Microarray and high-throughput sequencing technologies have been widely applied to identify functional miRNA, reliable target gene and have provided new insights into diagnosis and personalized treatment of many diseases[11].At present study, three miRNA expression profiles (GSE19943, GSE63492 and GSE116726), were downloaded from the GEO database (https://www.ncbi.nlm.nih.gov/geo/), to obtain miRNAs which are differentially expressed in IDD and normal discs (differentially expressed miRNAs, DEMs). Potential target genes of these DEMs were then predicted using online tools (TargetScan, miRDB, miRTarBase, and DIANA-TarBase). Subsequently, Gene Ontology (GO), Kyoto Encyclopedia of Genes and Genomes (KEGG) pathway enrichment analysis and protein-protein interaction (PPI) network analysis were employed to help us understand the molecular mechanisms underlying the progression of IDD. Finally, the expression levels of the DEMs in IDD and normal discs were determined by qRT-PCR. In conclusion, we identified and verified the key miRNAs and constructed miRNA-target genes regulatory network, which predict molecular mechanisms and provide new insights into the diagnosis and treatment of IDD.

\section{Materials And Methods}

\section{Collection of microarray datasets}

We identified datasets that compared the miRNA expression in IDD and normal discs from the GEO database. Then, three miRNA microarray datasets (GSE19943,GSE63492 and GSE116726) were obtained. The GSE19943 dataset based on the GPL9946 platform (Exiqon human miRCURY LNA ${ }^{\text {TM }}$ microRNA Array V11.0) included 3 IDD tissue and 3 normal discs tissue samples [12]. The GSE63492 dataset based on the GPL19449 platform (ExiqonmiRCURY LNA microRNA Array, 7th generation REV hsa, mmu, \&rno) contained 5 IDD tissue and 5 normal discs tissue samples [13, 14]. The GSE116726 dataset based on the GPL20712 platform (Agilent-070156 Human miRNA [miRNA version]) consisted of 3 IDD tissue and 3 normal discs tissue samples [15].

\section{Identification of differentially expressed miRNAs (DEMs)}

All raw microarray data were normalized and log2-transformed. Normalized data is shown in Fig.1. Then,we identified the differentially expressed miRNAs(DEMs) between degenerative and normal discs using the edgeR package in $R$ software[16]. We used $P$ value $<0.05$ and $\left|\log _{2} F C\right| \geq 0.5$ as cutoff criteria for screening DEMs. Venn diagrams were drawn by Venny 2.1 (http://bioinfogp.cnb.csic.es/tools/venny/).

\section{Prediction of target genes}

The online miRNA target prediction tools TargetScan (http://www.targetscan.org), miRDB (http://www.mirdb.org/), miRTarBase (http://mirtarbase.mbc.nctu.edu.tw), and DIANA-TarBase (http://diana.imis.athena-innovation.gr) were used to predict the target genes of the DEMs from GSE19943, GSE63492, and GSE116726. We only considered miRNA target genes that were predicted by at least two of the four tools. 


\section{Functional and pathway enrichment analysis}

Gene Ontology (GO) analysis[17] and Kyoto Encyclopedia of Genes and Genomes (KEGG) pathway analysis[18] of the predicted target genes were performed by an online tool, The database for annotation, visualization and integrated discovery (DAVID, http://david.ncifcrf.gov/)[19]. GO analysis describes our knowledge of the biological domain with respect to three aspects: biological processes (BP), cellular components (CC), and molecular functions (MF); KEGG is an integrated database resource for biological interpretation of genome sequences and other high-throughput data. $P$ value $<0.05$ was set as the cut-off criterion.

\section{PPI network and miRNA - target genes functional analysis network construction}

The Search Tool for the Retrieval of Interacting Genes (STRING; http://string-db.org/) [20]database is a pre-computed global resource, which was designed to predict functional associations between proteins. These target genes were mapped to STRING to evaluate the PPI information and then visualized with the Cytoscape software (http://www.cytoscape.org/). A combined score $>0.4$ was considered statistically significant. To screen the hub genes, node degree $\geq 10$ was set as the cut-off criterion. Moreover, functional and pathway enrichment analysis for target genes was performed using DAVID to create miRNA-target genes functionalanalysis network. The interaction network was visualized using Cytoscape software.

\section{Clinical Specimens}

The human lumber NP specimens(3 normal discs and 3 degenerative discs) were obtained from patients with lumbar disc herniation undergoing spinal surgery at our hospital. The severe degree of enrolled patients with IDD was assessed according to the Pfirrmann classification (normal: grade 1-2, degeneration: grade 3-5)[21].This study was approved by the Ethics Committees of the Yangpu Hospital Affiliated to Tongji University and all patients written informed consent before operation.

\section{RNA isolation and quantitative real-time PCR (qRT-PCR)}

Total RNA was isolated from NP samples following a previous study by Lee[22] and reverse transcribed into cDNA. The purity and concentration of RNAs was detected using A NanoDrop ND-1000 spectrophotometer(Thermo scientific, USA). After that, reverse transcription reaction was performed using miRcute miRNA RT Kit (TianGen, China). All qPCR reactions were performed in triplicate on a 7500 RealTime PCR System(Thermo Scientific, USA).U6 was used as the internal control. Relative expression levelswere analyzed with the standard curves method. The specific primers used were as follows:miR557,5'-GTTTGCACGGGTGGGCCTTGTCT-3'

(forward),5'-CTGTCAACGATACGCTACGTAACGGCATGACA-3'(reverse);miR-516a-3p,5'GGGCTGCTTCCTTTCAGAGGGT-3'(forward),5'-CTGTCAACGATACGCTACGTAACGGCATGACA3'(reverse);U6,5'-GTGCTCGCTTCGGCAGCACATATACTAAA-3'(forward), 5'CTGTCAACGATACGCTACGTAACGGCATGACA-3'(reverse). 


\section{Statistical analysis}

All data are presented as the means \pm SEM of three independent experiments. Statistical analysis were carried out using GraphPad prism 6.0 (GraphPad Software Inc.LaJolla,CA,USA).Differential expression levels of DEMs were compared by using Student's $t$ test. A two-tailed value of $P<0.05$ was considered as statistically significant.

\section{Results}

\section{Identification of DEMs and their target genes in IDD}

After standardization of the three microarray datasets, a total of 72,133 , and 1170 DEMs were identified in the GSE19943,GSE63492,and GSE116726 datasets(supplementary file 1,2 and 3). Further analysis identified miRNAs differentially regulated in IDD and normal discs as shown in the volcano plot (Fig.2A, 2B, 2C). Of these, 2 miRNAs, miR-516a-3p and miR-557, were found in all three datasets (Fig. 2D). 399 and 287 potential target genes were predicted for miR-516a-3p and miR-557, respectively, by using TargetScan, miRDB, miRTarBase, and DIANA-TarBase.

\section{GO and KEGG pathway enrichment analysis}

We used GO analysis to investigate the underlying biological function of these target genes predicted by DEMs. The top 30 of the $\mathrm{GO}$ enrichment analysis of target genes were shown in Fig. 3A. In the BP category, these target genes were mainly enriched in cell development, cell differentiation, positive regulation of the transforming growth factor beta (TGF-beta) receptor signaling pathway, and Notch receptor processing. In the MF category, these target genes were mainly enriched in $\mathrm{H} 4$ histone acetyltransferase activity, transmembrane receptor protein tyrosine phosphatase activity, R-SMAD binding, and histone acetyltransferase binding. Finally, of the top $30 \mathrm{GO}$ terms only Cul4-RING ubiquitin ligase complex related to a cellular component. The KEGG pathway analysis revealed that these target genes were enriched in the p53 signaling pathway, the Wnt signaling pathway and so on (Fig.3B).

\section{PPI network construction}

To further explore the molecular mechanisms of IDD, we mapped the target genes of the two DEMs onto the PPI network using the STRING database. Based on the information from this database, 267 PPI pairs were obtained as shown in Fig. 4. Then target genes from the PPI network with a node degree $\geq 10$ were selected as hub genes (Table 1). Of these genes, MAPK8 (degree, 23), BMP4 (degree, 20) and $G S K 3 B$ (degree, 19) were the nodes with the highest degrees. This suggests that MAPK8, BMP4 and GSK3B may be key targets correlated with IDD.

\section{miRNA - target genes functional analysis network construction and analysis}

The miRNA - target genes functional analysis network was constructed with the cytoscape software (Fig. 5A). We then investigated the potential interaction between miRNA and hub genes (Fig. 5B). The 
network showed that 9 hub genes (MAPK8, BMP4, GSK3B, CUL 1, STAT5B, UBE3A, LEF1, PAX6, and RPS6KB1) predicted to be regulated bymiR-577, While 4 hub genes (PTPN11, CBL, SMURF1, and SIAH1) predicted to be regulated by miR-516a-3p. Notably, the 3 highest degree hub genes (MAPK8, BMP4, and GSK3B) were modulated by miR-577. This network also showed that these hub genes were highly correlated with cell development, cell differentiation, and were mainly involved in the Wnt signaling pathway. Taken together, our results indicated that miR-577 and miR-516a-3p may be participate inthe Wnt signaling pathway to regulate intervertebral disc cells development, and differentiation through targeting these hub genes.

\section{Verification of DEMs}

The miR-577 andmiR-516a-3p expression lever were determined by qRT-PCR assay(Fig.6).

Compared with the normal disc tissue samples, miR-577 was decreased significantly in IDD tissue samples, and miR-516a-3p expression was increased significantly.

\section{Discussion}

IDD is a major cause of low back pain and various degenerative spinal disorders. IDD has been a global health issue, which places a heavy burden on the healthcare system and results in severe economic consequences [23]. To date, little is known about the molecular mechanisms and early diagnosis method of IDD. Thus, it is necessary to obtain a more comprehensive understanding of IDD progression.

miRNAs, a group of small non-coding RNAs, are regulators of target genes by altering mRNA translation or stability [3-5]. Emerging evidence indicates that miRNAs play a key role in the development and progression of IDD [6, 7], and may be effective therapeutic targets [9] and biomarkers[8]. Recent advances in microarray techniques enable us to determine the functional miRNAs and target genes in the progression of diseases, which can be used to identify key miRNAs for diagnosis and therapy of IDD.

In this study,3 miRNA microarray datasets were downloaded from GEO to obtain DEMs between normal and degenerative disc samples. Two DEMs, miR-557 and miR-516a-3p, were present in all the datasets. In the three microarray datasets, miR-557 was down-regulated. miR-516a-3p was down-regulated in datasets. However, it was up-regulated in GSE116726 dataset. Our qRT-PCR data showed that miR-577 was significantly downregulated,and miR-516a-3p was significantly upregulated,which consistent with the GSE116726 dataset.MiR-557 is a recently identified miRNA. Previous studies have been reported that it is related to the progression of various human cancers, such as lung cancer, gastric cancer, hepatocellular carcinoma and breast cancer [24-27]. Qiu et al. found that miR-557 can suppress the proliferation and invasion of lung cancer cells by negatively regulating the expression of LEF1 [24], which corresponded to our analysis.Yu et al. studies have shown that miR-577 could be a marker for the diagnosis andprognosis of gastric cancer[25]. Regarding miR-516a-3p, there are only two publications in PubMed. The studies indicated that miR-516a-3p can regulate the metastasis and progression of 
tumor,and influence chemosensitivity[28, 29].These findings might suppose that miR-577 andmiR-516a$3 p$ may be potential key regulators in the development of IDD.

Our GO analysis showed that the target genes were mainly associated with cell development and cell differentiation functions. The current lack of early-stage diagnosis and treatment for IDD is associated with a poor understanding of the biological mechanisms that regulate intervertebral disc (IVD) development and degeneration [30]. Intervertebral discs consist of a nucleus pulposus (NP), an annulus fibrosus (AF) and cartilage endplates (CEP). NP cells play critical roles in maintaining the integrity of intervertebral discs by secreting a complex extracellular matrix (ECM)[31]. Understanding the mechanism of NP development will provide the foundation for future repair, regeneration, and tissue engineering strategies for IVD disease [32]. Moreover, KEGG pathway analysis showed that most significant pathways were classical signaling pathways, such as the p53 signaling pathway and the Wnt signaling pathway. p53 is a famous tumor suppressor and plays key roles in metabolism. Xiong et al. found that increasing p53 levels could improve the negative effects of NP cell viability and integrity under low glucose conditions[33]. The Wnt signaling pathway is a major signaling pathway that participates in IDD progression. Activating Wnt/ $\beta$-catenin signaling could regulates aggrecan expression,and induces expression of matrix metalloproteinases and TGF- $\beta$ in NP cells, thereby promoting cellular senescence and IVD degeneration[34]. Previous studies have reported that miRNA can modulate intervertebral disc NP cells apoptosis, senescence, proliferation, and ECM synthesis via Wnt signaling [35-37]. All of these research findings are consistent with our results.

By constructing a PPI network, we identified 13 hub genes with degrees $\geq 10$, including MAPK8, BMP4, and GSK3B, which were the 3 nodes with the highest degrees. To analyze the potential role of hub genes, an miRNA-target gene GO and KEGG analysis network was created and it was found that the hub genes, MAPK8, BMP4 and GSK3B, were mainly modulated by miR-577. Further analysis indicated that the hub genes were significantly associated with cell development, cell differentiation, and the Wnt signaling pathway. A literature search showed that the interaction among IDD and hub genes has rarely been reported. MAPK8, which is also known as JNK1, is a member of the MAP kinase and JNK family. Its functions include cell proliferation, cell differentiation, and apoptosis [38, 39]. Hua et al.found that MAPK8 might be candidate biomarker for apoptosis in Melanosis coli [40]. Wang et al. verified that MAPK8 regulates the differentiation of embryonic stem cells by inhibiting/activating JNK signaling [41]. Furthermore, Ren et al.conclude that enhanced expressions of miR-330 inhibits atherosclerotic plaqueformation while promoting vascular endothelial cell proliferation by targeting MAPK8 via the Wnt signaling pathway in acute coronary syndrome rats [42]. Thus, MAPK8 might regulate the process of IDD. BMP4 is a member of the bone morphogenetic protein (BMP) family which belongs to the TGF-beta superfamily. It has been reported that BMPs play key roles in development of human diseases, and in maintenance of tissue homeostasis[43]. From the network, we found that BMP4 is only involved in the regulation of cell development, cell differentiation and the TGF-beta signaling pathway. Data from ElSafadi et al. suggests the existence of reciprocal relationship between TGF-beta and BMP signaling that regulates hBMSC lineage commitment and differentiation [44]. In addition, some research has shown that TGF-beta, Wnt signaling pathway components and BMP4 are essential genes during the notochord to NP 
transition $[45,46]$. Therefore, BMP4 maybe closely involved with development of NP cells. GSK3B is an integral kinase within the $\beta$-catenin destruction complex. GSK3B can suppress Wnt signaling by phosphorylation and proteasomal degradation of $\beta$-catenin[47]. Several studies have shown modifications of GSK3B leading to Wnt/ $\beta$-catenin signaling activation/suppression, which promotes cancer progression and chemosensitivity $[48,49]$. Importantly, Wang et al. found that circSEMA4Bcanacts as a miRNA sponge and competes with GSK-3 $\beta$ for binding to miR-431, thereby inhibiting IL-1 $\beta$-induced degeneration in NPCsvia regulating Wnt signaling[50]. The results of these previous studies were in agreement with our study, which showed that GSK3B through modulation of Wnt signaling pathway activity might affect the development of IDD.

In conclusion, this study identified two novel DEMs (miR-557 and miR-516a-3p) and 13 hub genes via a comprehensive bioinformatics analysis approach. Validation experiments by qRT-PCR suggested that miR-557 and miR-516a-3p may have a certain influence on the pathological processes of IDD. Notably, the roles of miR-557 through its targeting of MAPK8, BMP4 and GSK3B may improve our understanding and provide new therapeutic targets for IDD. However, there are still some deficiencies in this study. First, the sample sizes of microarray data are small, so further studies with larger sample sizes are still needed in order to confirm the results. second, only the top three hub genes and Wnt signaling were selected for further analysis, which might overlook important functions of other hub genes and pathways.

\section{Declarations}

\section{Acknowledgements}

Not applicable.

\section{Funding}

Not applicable.

\section{Availability of data and material}

The microarray datasets used and/or analysed during the current study are all in the manuscript.

\section{Conflict of interest}

All the authors declare no conflicts of interest.

\section{Author contributions}

Conception and design: Xiaodong Liu and Hongze Chang.

Collection and assembly of data: Feng Cai, Yan Zhang, Liang Liu, Hui Liu.

Data analysis and interpretation: Hongze Chang, Xiaolong Yang, Kemin You, Mingwei Jiang 
Manuscript writing: Xiaodong Liu and Hongze Chang.

Final approval of manuscript: All authors

\section{References}

1. Freemont AJ: The cellular pathobiology of the degenerate intervertebral disc and discogenic back pain. Rheumatology (Oxford) 2009, 48(1):5-10.

2. Wang F, Cai F, Shi R, Wang XH, Wu XT: Aging and age related stresses: a senescence mechanism of intervertebral disc degeneration. Osteoarthritis Cartilage 2016, 24(3):398-408.

3. Ambros V: The functions of animal microRNAs. Nature 2004, 431(7006):350-355.

4. Bartel DP: MicroRNAs: genomics, biogenesis, mechanism, and function. Cel/ 2004, 116(2):281-297.

5. Calin GA, Croce CM: MicroRNA signatures in human cancers. Nat Rev Cancer 2006, 6(11):857-866.

6. Tao B, Yi J, Huang C, Xu W, Qin C, Chen L, Chen J, Gao Y, Wang R: microRNA96 regulates the proliferation of nucleus pulposus cells by targeting ARID2/AKT signaling. Molecular medicine reports 2017, 16(5):7553-7560.

7. Tan $\mathrm{H}$, Zhao L, Song R, Liu Y, Wang L: microRNA-665 promotes the proliferation and matrix degradation of nucleus pulposus through targeting GDF5 in intervertebral disc degeneration. Journal of cellular biochemistry 2018, 119(9):7218-7225.

8. Ye D, Dai L, Yao Y, Qin S, Xie H, Wang W, Liang W: miR-155 Inhibits Nucleus Pulposus Cells' Degeneration through Targeting ERK 1/2. Disease markers 2016, 2016:6984270.

9. Ji ML, Jiang H, Zhang XJ, Shi PL, Li C, Wu H, Wu XT, Wang YT, Wang C, Lu J: Preclinical development of a microRNA-based therapy for intervertebral disc degeneration. Nature communications 2018, 9(1):5051.

10. Penolazzi L, Lambertini E, Bergamin LS, Roncada T, De Bonis P, Cavallo M, Piva R: MicroRNA-221 silencing attenuates the degenerated phenotype of intervertebral disc cells. Aging 2018, 10(8):20012015.

11. Li MH, Fu SB, Xiao HS: Genome-wide analysis of microRNA and mRNA expression signatures in cancer. Acta pharmacologica Sinica 2015, 36(10):1200-1211.

12. Wang HQ, Yu XD, Liu ZH, Cheng X, Samartzis D, Jia LT, Wu SX, Huang J, Chen J, Luo ZJ: Deregulated miR-155 promotes Fas-mediated apoptosis in human intervertebral disc degeneration by targeting FADD and caspase-3. The Journal of pathology 2011, 225(2):232-242.

13. Liu X, Che L, Xie YK, Hu QJ, Ma CJ, Pei YJ, Wu ZG, Liu ZH, Fan LY, Wang HQ: Noncoding RNAs in human intervertebral disc degeneration: An integrated microarray study. Genomics data 2015, 5:8081.

14. Lan PH, Liu ZH, Pei YJ, Wu ZG, Yu Y, Yang YF, Liu X, Che L, Ma CJ, Xie YK et al: Landscape of RNAs in human lumbar disc degeneration. Oncotarget 2016, 7(39):63166-63176. 
15. Wang H, He P, Pan H, Long J, Wang J, Li Z, Liu H, Jiang W, Zheng Z: Circular RNA circ-4099 is induced by TNF-alpha and regulates ECM synthesis by blocking miR-616-5p inhibition of Sox9 in intervertebral disc degeneration. Experimental \& molecular medicine 2018, 50(4):27.

16. Ritchie ME, Phipson B, Wu D, Hu Y, Law CW, Shi W, Smyth GK: limma powers differential expression analyses for RNA-sequencing and microarray studies. Nucleic acids research 2015, 43(7):e47.

17. The Gene Ontology (GO) project in 2006. Nucleic acids research 2006, 34(Database issue):D322-326.

18. Kanehisa M: The KEGG database. Novartis Foundation symposium 2002, 247:91-101; discussion 101-103, 119-128, 244-152.

19. Huang da W, Sherman BT, Lempicki RA: Systematic and integrative analysis of large gene lists using DAVID bioinformatics resources. Nature protocols 2009, 4(1):44-57.

20. von Mering C, Huynen M, Jaeggi D, Schmidt S, Bork P, Snel B: STRING: a database of predicted functional associations between proteins. Nucleic acids research 2003, 31(1):258-261.

21. Pfirrmann CW, Metzdorf A, Zanetti M, Hodler J, Boos N: Magnetic resonance classification of lumbar intervertebral disc degeneration. Spine 2001, 26(17):1873-1878.

22. Lee JTY, Cheung KMC, Leung VYL: Extraction of RNA from tough tissues with high proteoglycan content by cryosection, second phase separation and high salt precipitation. J Biol Methods 2015, 2(2).

23. Martin BI, Deyo RA, Mirza SK, Turner JA, Comstock BA, Hollingworth W, Sullivan SD: Expenditures and health status among adults with back and neck problems. Jama 2008, 299(6):656-664.

24. Qiu J, Hao Y, Huang S, Ma Y, Li X, Li D, Mao Y: MiR-557 works as a tumor suppressor in human lung cancers by negatively regulating LEF1 expression. Tumour biology : the journal of the International Society for Oncodevelopmental Biology and Medicine 2017, 39(6):1010428317709467.

25. Yu Z, Zhang W, Deng F: MicroRNA-577 inhibits gastric cancer growth by targeting E2F transcription factor 3. Oncology letters 2015, 10(3):1447-1452.

26. Katayama Y, Maeda M, Miyaguchi K, Nemoto S, Yasen M, Tanaka S, Mizushima H, Fukuoka Y, Arii S, Tanaka H: Identification of pathogenesis-related microRNAs in hepatocellular carcinoma by expression profiling. Oncology letters 2012, 4(4):817-823.

27. Razaviyan J, Hadavi R, Tavakoli R, Kamani F, Paknejad M, Mohammadi-Yeganeh S: Expression of miRNAs Targeting mTOR and S6K1 Genes of mTOR Signaling Pathway Including miR-96, miR-557, and miR-3182 in Triple-Negative Breast Cancer. Applied biochemistry and biotechnology 2018, 186(4):1074-1089.

28. Takei $Y$, Takigahira M, Mihara K, Tarumi Y, Yanagihara K: The metastasis-associated microRNA miR$516 a-3 p$ is a novel therapeutic target for inhibiting peritoneal dissemination of human scirrhous gastric cancer. Cancer research 2011, 71(4):1442-1453.

29. Zhang H, Lian Z, Sun G, Liu R, Xu Y: Loss of miR-516a-3p mediates upregulation of ABCC5 in prostate cancer and drives its progression. OncoTargets and therapy 2018, 11:3853-3867. 
30. McCann MR, Seguin CA: Notochord Cells in Intervertebral Disc Development and Degeneration. Journal of developmental biology 2016, 4(1).

31. Chen J, Xie JJ, Jin MY, Gu YT, Wu CC, Guo WJ, Yan YZ, Zhang ZJ, Wang JL, Zhang XL et al: Sirt6 overexpression suppresses senescence and apoptosis of nucleus pulposus cells by inducing autophagy in a model of intervertebral disc degeneration. Cell death \& disease 2018, 9(2):56.

32. Alkhatib B, Ban GI, Williams S, Serra R: IVD Development: Nucleus pulposus development and sclerotome specification. Current molecular biology reports 2018, 4(3):132-141.

33. Xiong X, Dai L, Liang W, Zhang J, Qin S, Cao W, Ye D, Liang P, Liu Z: Protective effect of p53 on the viability of intervertebral disc nucleus pulposus cells under low glucose condition. Biochemical and biophysical research communications 2017, 490(4):1414-1419.

34. Hiyama A, Sakai D, Risbud MV, Tanaka M, Arai F, Abe K, Mochida J: Enhancement of intervertebral disc cell senescence by WNT/beta-catenin signaling-induced matrix metalloproteinase expression. Arthritis and rheumatism 2010, 62(10):3036-3047.

35. Sun Z, Jian Y, Fu H, Li B: MiR-532 downregulation of the Wnt/beta-catenin signaling via targeting Bcl9 and induced human intervertebral disc nucleus pulposus cells apoptosis. Journal of pharmacological sciences 2018, 138(4):263-270.

36. Wang X, Zou M, Li J, Wang B, Zhang Q, Liu F, Lu G: LncRNA H19 targets miR-22 to modulate H2 02 induced deregulation in nucleus pulposus cell senescence, proliferation, and ECM synthesis through Wnt signaling. Journal of cellular biochemistry 2018, 119(6):4990-5002.

37. Yang S, Li L, Zhu L, Zhang C, Li Z, Guo Y, Nie Y, Luo Z: Bu-Shen-Huo-Xue-Fang (BSHXF) modulates nucleus pulposus cell proliferation and extracellular matrix (ECM) remodeling in intervertebral disc degeneration through miR-483 regulation of Wnt pathway. Journal of cellular biochemistry 2018.

38. Tournier C, Hess P, Yang DD, Xu J, Turner TK, Nimnual A, Bar-Sagi D, Jones SN, Flavell RA, Davis RJ: Requirement of JNK for stress-induced activation of the cytochrome c-mediated death pathway. Science (New York, NY) 2000, 288(5467):870-874.

39. Kim SW, Muise AM, Lyons PJ, Ro HS: Regulation of adipogenesis by a transcriptional repressor that modulates MAPK activation. The Journal of biological chemistry 2001, 276(13):10199-10206.

40. Hua $X$, Chen J, Wu L: Identification of candidate biomarkers associated with apoptosis in melanosis coli: GNG5, LPAR3, MAPK8, and PSMC6. Bioscience reports 2019, 39(1).

41. Wang Y, Bi Y, Zuo Q, Zhang W, Li D, He NN, Cheng S, Zhang YN, Li B: MAPK8 regulates chicken male germ cell differentiation through JNK signaling pathway. Journal of cellular biochemistry 2018 , 119(2):1548-1557.

42. Ren J, Ma R, Zhang ZB, Li Y, Lei P, Men JL: Effects of microRNA-330 on vulnerable atherosclerotic plaques formation and vascular endothelial cell proliferation through the WNT signaling pathway in acute coronary syndrome. Journal of cellular biochemistry 2018, 119(6):4514-4527.

43. Wang RN, Green J, Wang Z, Deng Y, Qiao M, Peabody M, Zhang Q, Ye J, Yan Z, Denduluri S et al: Bone Morphogenetic Protein (BMP) signaling in development and human diseases. Genes \& diseases 2014, 1(1):87-105. 
44. El-Safadi M, Shinwari T, Al-Malki S, Manikandan M, Mahmood A, Aldahmash A, Alfayez M, Kassem $\mathrm{M}$, Alajez NM: Convergence of TGFbeta and BMP signaling in regulating human bone marrow stromal cell differentiation. Scientific reports 2019, 9(1):4977.

45. Peck SH, McKee KK, Tobias JW, Malhotra NR, Harfe BD, Smith LJ: Whole Transcriptome Analysis of Notochord-Derived Cells during Embryonic Formation of the Nucleus Pulposus. Scientific reports 2017, 7(1):10504.

46. Tang R, Jing L, Willard VP, Wu CL, Guilak F, Chen J, Setton LA: Differentiation of human induced pluripotent stem cells into nucleus pulposus-like cells. Stem cell research \& therapy 2018, 9(1):61.

47. van Noort M, Meeldijk J, van der Zee R, Destree $\mathrm{O}$, Clevers $\mathrm{H}$ : Wnt signaling controls the phosphorylation status of beta-catenin. The Journal of biological chemistry 2002, 277(20):1790117905.

48. Zhu X, Li Y, Zhao S, Zhao S: LSINCT5 activates Wnt/beta-catenin signaling by interacting with NCYM to promote bladder cancer progression. Biochemical and biophysical research communications 2018, 502(3):299-306.

49. Jiang C, He C, Wu Z, Li F, Xiao J: Histone methyltransferase SETD2 regulates osteosarcoma cell growth and chemosensitivity by suppressing Wnt/beta-catenin signaling. Biochemical and biophysical research communications 2018, 502(3):382-388.

50. Song J, Wang HL, Song KH, Ding ZW, Wang HL, Ma XS, Lu FZ, Xia XL, Wang YW, Fei Z et al: CircularRNA_104670 plays a critical role in intervertebral disc degeneration by functioning as a ceRNA. Experimental \& molecular medicine 2018, 50(8):94.

\section{Table}

Table 1 Hub genes identifed in the PPI network

\begin{tabular}{lclclc}
\hline Gene symbol & Degree & Gene symbol & Degree & Gene symbol & Degree \\
\hline MAPK8 & 23 & UBE3A & 13 & SMURF1 & 10 \\
BMP4 & 20 & LEF1 & 12 & SIAH1 & 10 \\
GSK3B & 19 & PTPN11 & 11 & RPS6KB1 & 10 \\
& & & & & \\
CUL1 & 15 & PAX6 & 11 & & \\
& & & & & \\
STAT5B & 13 & CBL & 11 & & \\
\hline
\end{tabular}

\section{Figures}




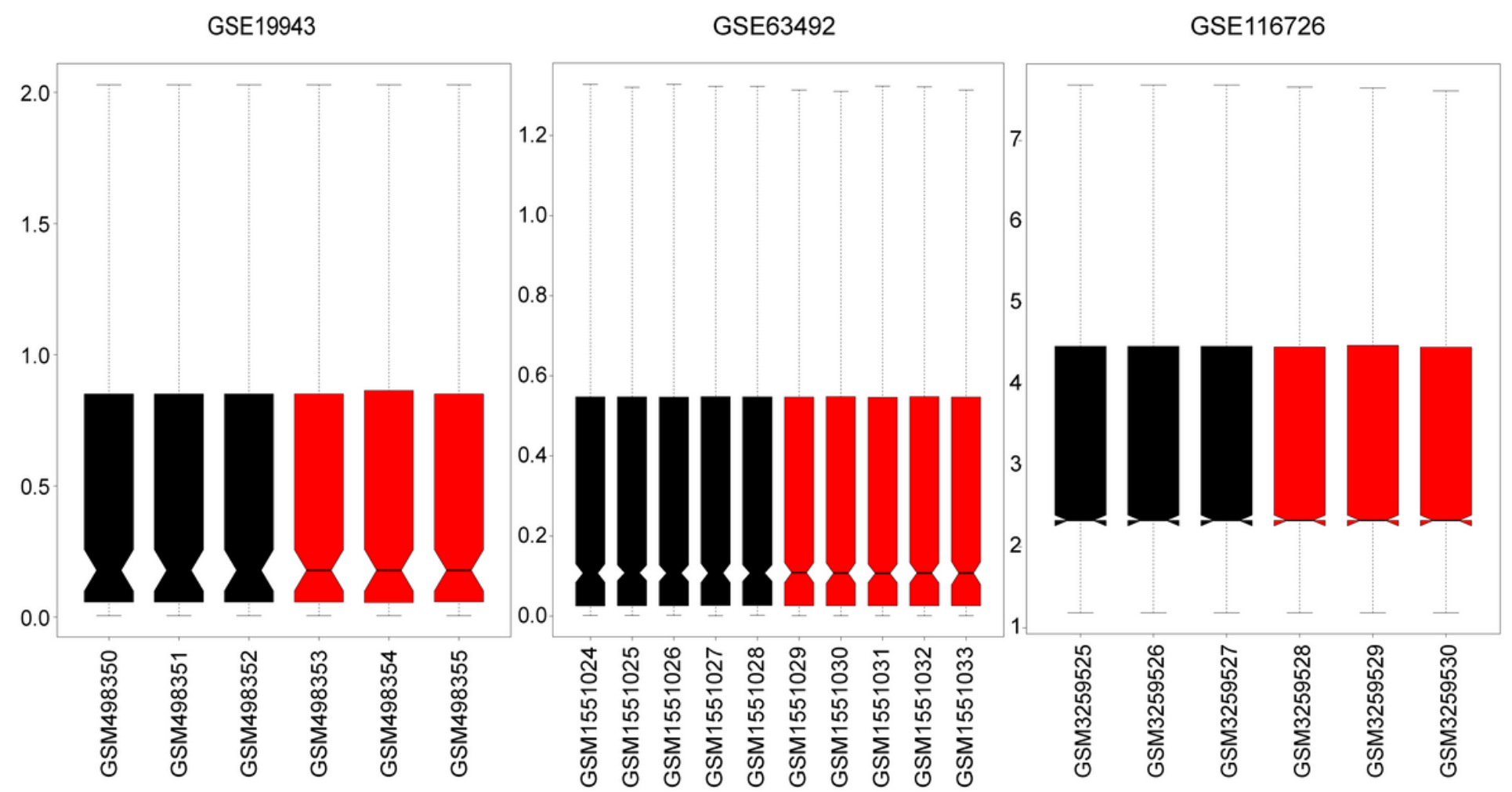

Figure 1

Datas after normalization of GSE19943 , GSE63492 and GSE116726. 

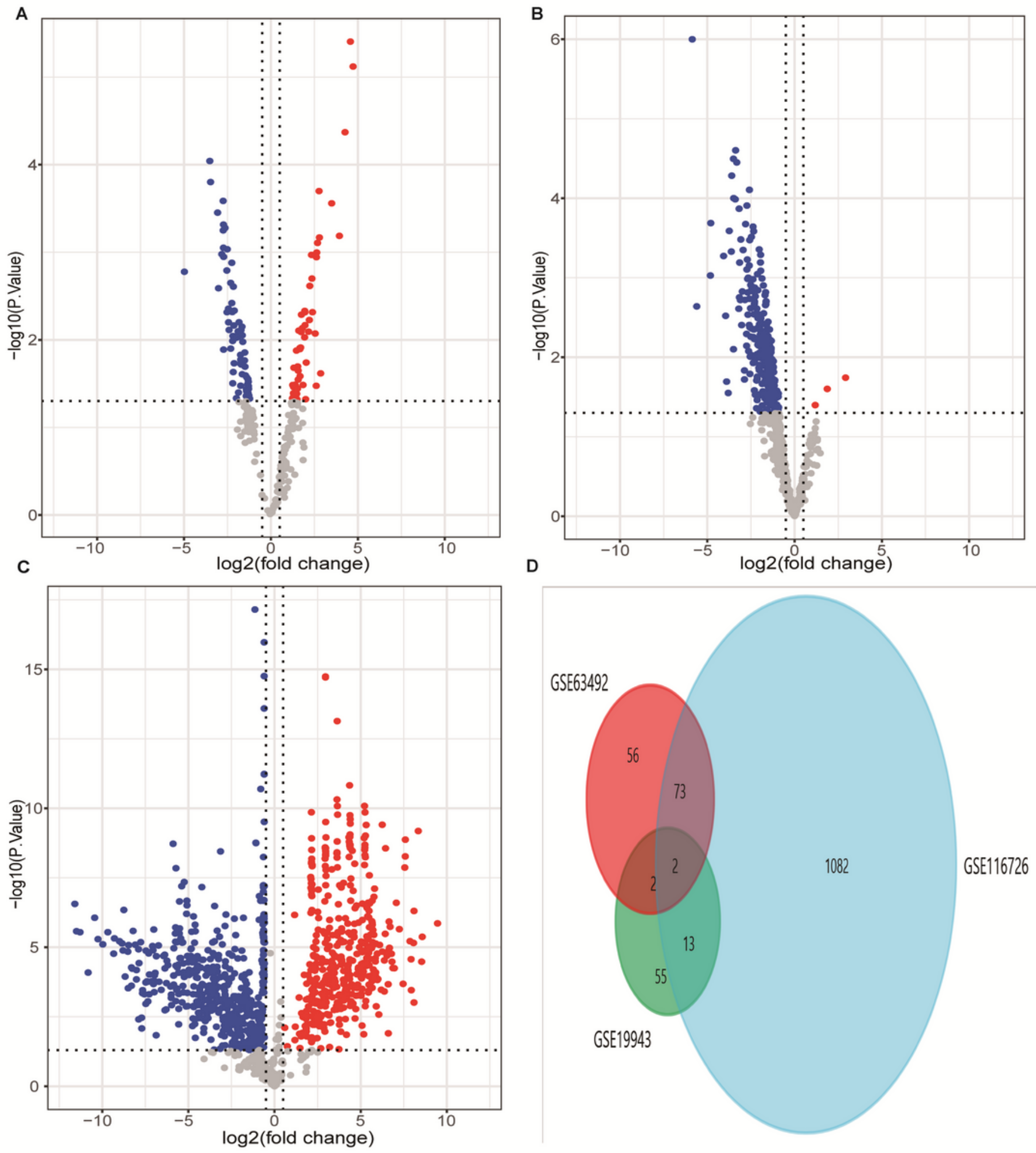

\section{Figure 2}

A:Volcano plot displaying the DEMs of GSE19943.B:Volcano plot displaying the DEMs of GSE63492. C:Volcano plot displaying the DEMs of GSE116726.d. DEMs were selected with a $\| \log 2 \mathrm{FC} \mid \geq 0.5$ and $P$ value $<0.05$ among the miRNA expression profiling datasets GSE19943, GSE63492 and GSE116726. The 3 datasets showed an overlap of 2 miRNAs. The grey dots represent miRNAs that are not differentially expressed between IDD tissue samples and normal disc tissue samples, the blue dots represent the 
downregulated miRNAs, and red dots represent the upregulated miRNAs. DEMs, differentially expressed miRNAs. FC, fold change.

A

Top 30 of GO Enrichment

type $B$ pancreatic cell differentiationtype $B$ pancreatic cell developmenttransmembrane receptor protein tyrosine phosphatase activityregulation of transforming growth factor beta receptor signaling pathway regulation of keratinocyte proliferation-" regulation of cardiac muscle hypertrophy- . R-SMAD binding protein homotrimerization positive regulation of transforming growth factor beta receptor signalpositive regulation of smooth muscle contractionpositive regulation of osteoclast differentiationNotch receptor processingnegative regulation of myoblast differentiationmembrane depolarization during action potential $\bullet$ histone acetyltransferase binding $\mathrm{H} 4$ histone acetyltransferase activity glycogen catabolic process glandular epithelial cell differentiationglandular epithelial cell developmententeroendocrine cell differentiationembryonic placenta morphogenesiscyclic-nucleotide phosphodiesterase activity Cul4-RING ubiquitin ligase complexcolumnar/cuboidal epithelial cell developmentcerebellar Purkinje cell layer morphogenesiscerebellar Purkinje cell layer developmentcerebellar cortex morphogenesiscerebellar cortex formationcellular response to epidermal growth factor stimulus 3',5'-cyclic-nucleotide phosphodiesterase activity 4567889 enrich factor

.
B

Top 30 of Pathway Enrichment

diff_gene_count

- 4

- 6

- 8

10

12

14

16

pvalue

0.008

0.006

0.004

0.002

GO_domain

- biological_process

A cellular_component

- molecular_function

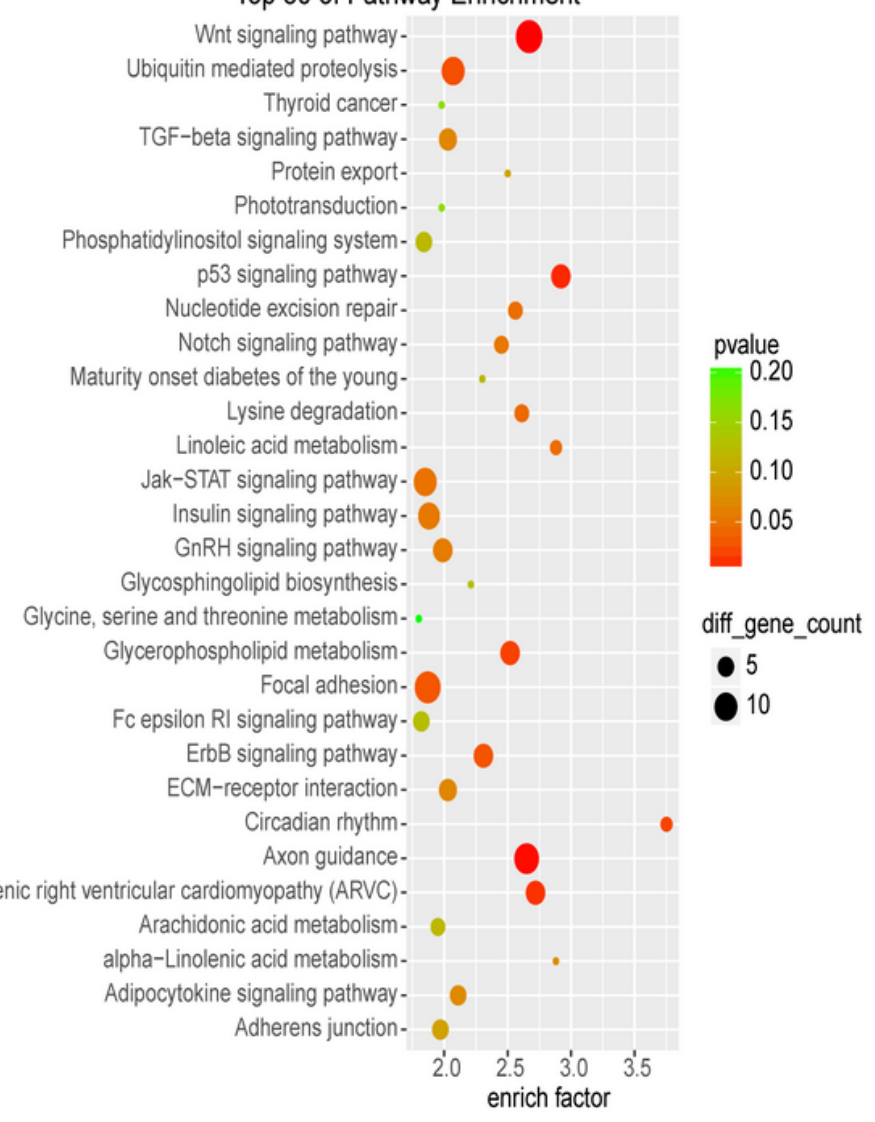

\section{Figure 3}

\section{A: Top 30 terms of GO enrichment.B: Top 30 of KEGG pathway enrichment.The circles represent biological process; the triangles represent cell component; the squares represent molecular function.}




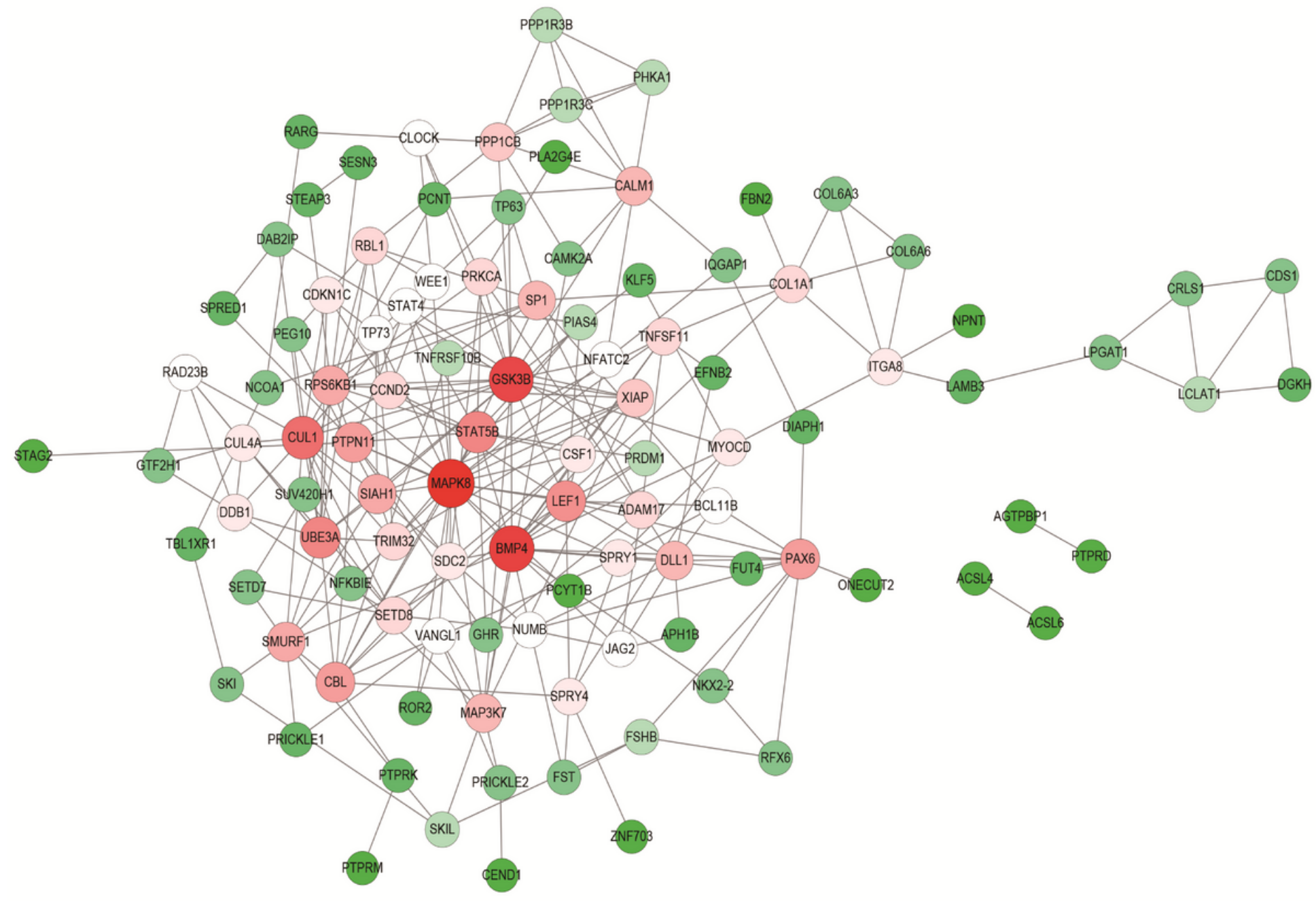

\section{Figure 4}

Protein-protein interaction(PPI) network of target genes. All nodes with a combined interaction score of $>0.4$ are displayed. The oval represent the target genes; The color of Node from green to red indicating a change in the node degree. 
A



B

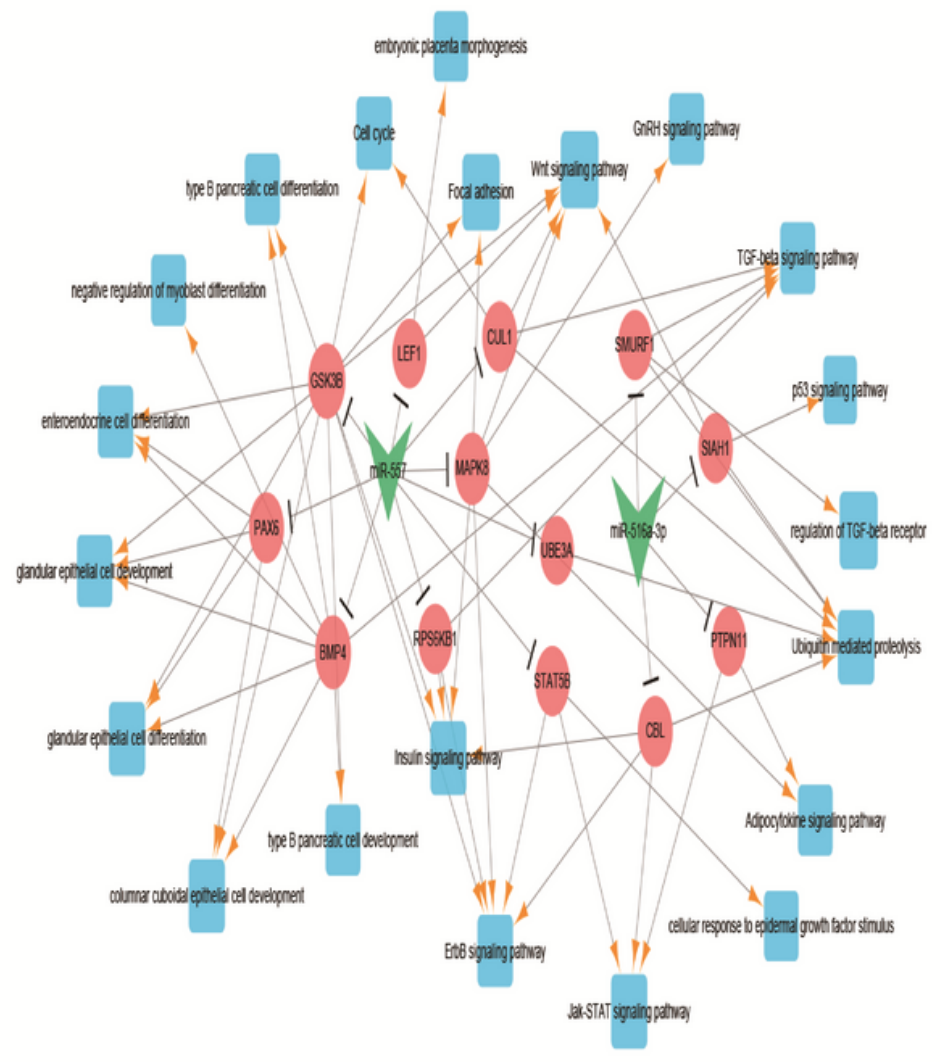

\section{Figure 5}

A:The regulatory network between miRNAs, target genes and functional analysis.B: The regulatory network between miRNAs, hub genes and functional analysis. In the network,green diamond nodes represent DEMs; red oval nodes represent the target genes; blue rectangle represent the GO and KEGG pathway enrichment. Edge target arrow shape represent interaction. vertical indicate negative correlation and arrow represent positive correlation. 




Figure 6

The relative expression level of miR-516a-3p and miR-557 between normal disc tissues ( $n=3)$ and IDD tissue samples $(n=3)$ by qRT-PCR. ${ }^{*} P<0.05$.

\section{Supplementary Files}

This is a list of supplementary files associated with this preprint. Click to download.

- GSE19943DiffSigmiRNAandexprssiondata.xlsx

- GSE63492DiffSigmiRNAandexprssiondata.xlsx

- GSE116726DiffSigmiRNAandexprssiondata.xlsx 